\title{
Başarısız kalça revizyon cerrahisi sonrası tedavi seçenekleri
}

\author{
Treatment options after failed hip revision surgery
}

\author{
Hakan Kocaoğlu
}

Ankara Üniversitesi Tıp Fakültesi, Ortopedi ve Travmatoloji Ana Bilim Dalı, Ankara

Kalça artroplasti cerrahisindeki yeni gelişmelerle birlikte, ameliyat sonuçlarındaki başarı da son zamanlarda giderek artmaktadır. Kalça artroplastisinde ikincil revizyonlar, teknik olarak ciddi zorluklar barındıran ve elde edilen sonuçların çoğu zaman hasta beklentileri ile yeterince örtüşmediği ameliyatlardır. Cerrahi teknik ve implant tasarımlarındaki iyileştirmeler ve hasta riskinin ameliyat öncesi değerlendirilmesi gibi birçok atılıma rağmen, başarısız revizyon cerrahileri halen varlığını sürdürmektedir. Bu olguların tedavilerinde standart bir yol izlemek pek mümkün değildir ve her olguya özel olarak bir yaklaşım geliştirmek gereklidir. En sık karşılaşılan başarısızlık nedeni olan, tekrarlayan çıkıkları engellemek için; kısıtlı (constrained) sistemler, büyük femoral baş kullanımı ya da dual mobility eklem seçenekleri yaygın olarak kullanılmaktadır. Asetabulumda kemik defekti bulunan olgularda 'jumbo' kaplar, metal kafesler, konik (cone) ya da LUMIC ${ }^{\circledR}$ tipi asetabular sistemler, trabeküler metal veya özel üretim implantlar; femur için gerekli olduğu durumlarda da tümör protezleri ya da total femur gibi seçenekler de kullanılabilmektedir.

Anahtar sözcükler: revizyon kalça artroplastisi; mekanik gevşeme; periprostetik enfeksiyon; kemik kaybı
With the new developments in hip arthroplasty surgery, the success in surgical results has been increasing recently. Secondary revisions in hip arthroplasty are surgeries that have serious technical difficulties and the results obtained often do not adequately meet the expectations of the patient. Despite many breakthroughs such as improvements in surgical techniques and implant designs, and preoperative assessment of patient risk, there still exist unsuccessful revision surgeries. It is not possible to follow a standard method in the treatment of these cases, and it is necessary to develop a special approach for each case. Constrained systems, large femoral heads, or dual mobility cups are commonly used to prevent recurrent dislocations which are the most common causes of failure. Jumbo cups, metal cages, cone or LUMIC ${ }^{\circledR}$ type acetabular systems, trabecular metal or custom-made implants can be used in cases with bone defects in the acetabulum, and where it is necessary for the femur, options such as tumor prostheses or total femur may also be utilized.

Key words: revision hip arthroplasty; mechanical loosening; periprosthetic infection; bone loss
K alça artroplasti cerrahisindeki yeni gelişmelerle birlikte, ameliyat sonuçlarındaki başarı da son zamanlarda giderek artmaktadır. Güncel verilere göre, 2030'da primer total kalça artroplastisi (TKA) insidansının 15 yı önceye göre \%174 artarak, Amerika Birleşik Devletleri'nde yılda yaklaşık 572.000 olguya ulaşacağı tahmin edilmektedir.[1] Bu artışa bağlı olarak, revizyon TKA ve ikincil revizyon yükünün de önemli ölçüde artacağı öngörülebilir. Hâlihazırda, Amerika Birleşik Devletleri'nde gerçekleştirilen tüm kalça artroplastilerinin yaklaşık \%18'i revizyon girişimleridir. Bu da yıllık olarak yaklaşık 50.000 ameliyat anlamına gelir. ${ }^{[2-4]}$

Kalça artroplastisinde ikincil revizyonlar, teknik olarak ciddi zorluklar barındıran ve elde edilen sonuçların çoğu zaman hasta beklentileri ile yeterince örtüşmediği ameliyatlardır. Genç ve daha aktif popülasyonda kalça artroplastisi sıklığı arttıkça, birden çok revizyon geçirmiş hastaların sayısı da artış gösterecektir. ${ }^{[4]}$ Bazı serilerde, bu grup hastalardaki bir yıllık protez sağkalımının \%54 gibi düşük oranlarda olduğu görülmektedir. ${ }^{[1]}$

Revizyon kalça artroplastisinin başarısızlık nedenlerinin aydınlatılması, olası çoklu revizyon olgularının önüne geçeceği gibi, medikal ve ekonomik yükün de azaltılması açısından çok önemlidir. Primer veya revizyon artroplasti cerrahisinde başarısızlık, genellikle hasta ve cerrahi ile ilgili birçok etkenin bir araya gelmesiyle ortaya çıkar. Yine de, revizyon nedenleri ayrı ayrı incelendiğinde, en sık etkenin çıkık ve mekanik gevşeme olduğu görülür. ${ }^{[5]}$

İletişim / Contact: Doç. Dr. Hakan Kocaoğlu • E-posta / E-mail: kocaoglu@ankara.edu.tr

ORCID iD: Hakan Kocaoğlu, 0000-0002-1421-3555

Geliş / Received: 22 Eylül 2021 • Kabul / Accepted: 1 Ekim 2021 
Revizyon kalça artroplastisi sonrasında çıkık riski, primer cerrahiye göre yaklaşık dört kat daha yüksektir. $^{[6]}$ Çıkık ve instabilite tüm re-revizyon nedenlerinin \%35'ini oluşturmaktadır. ${ }^{[7-9]}$ Toplam revizyon TKA olgularının \%30'dan fazlasını 75 ila 84 yaşındaki hastalar oluşturur. Bu alt grupta kalça artroplastisinin en sık revizyon nedeni ise periprostetik kırıktır. Ileri yaş, hareketsizlik ve uzun süreli tedavisiz osteoporoz, çok düşük enerjili yaralanmalarla bile büyük periprostetik kırıkların oluşmasına yol açabilmektedir. ${ }^{[10]}$

Bu iki sebebe ek olarak, artroplasti sonrası periprostetik enfeksiyon da en korkulan komplikasyonlardandır. Primer cerrahinin uzamış ameliyat süresi, hastanın komorbidite yükü, obezite, romatoid artrit, ameliyat öncesi sigara kullanımı ve önceki kırık cerrahisi, kalça artroplastisi sonrası görülen periprostetik enfeksiyon için daha bilinen risk faktörleridir. ${ }^{[7]}$

Periprostetik enfeksiyonun tek ya da iki basamaklı cerrahisi sonrasında, hem yumuşak dokuda hem de kemikte ciddi defektler meydana gelebilir. Olgular, enfeksiyon tedavisinin sonunda hem çıkık hem de rekonstrüksiyona yetecek kemik stok kalmayacak şekilde başarısızlık ile sonuçlanabilir. ${ }^{[11,12]}$

\section{TEDAVI SEÇENEKLERi}

Özellikle çok nadir görülen bu olguların tedavilerinde, standart bir yol izlemek pek mümkün değildir. Genelde, her olguya özel bir yaklaşım geliştirmek gereklidir. Başarısızlık, altta yatan birçok etkenin birleşmesi sonucu olmuş olabilir ve cerrahi planlama yapılmadan önce her bir etken aydınlatılmalıdır. Özellikle, pelvik bölgede görülen kemik kayıplarının değerlendirilmesi için bilgisayarlı tomografi gibi kesitsel görüntülemeler mutlaka yapılmalıdır.

En sık karşılaşılan başarısızlık nedeni olan tekrarlayan çıkıkları engellemek için, kısıtlı (constrained) sistemler, büyük femoral baş kullanımı ya da dual mobility eklem seçenekleri yaygın olarak kullanılmaktadır. ${ }^{[13-15]}$

Kısıtlı bileşenlerin mantığı, femur başını bir polietilen liner içine sıkıştırarak ve kalça hareket açıklığını kısıtlayarak instabiliteyi önlemektir. ${ }^{[6]}$ Bununla birlikte, böyle bir mekanizma, kemik-implant ara yüzüne uygulanan yüksek stresle sonuçlanacak, bu da linerda gevşemeye yol açarak kilitleme halkası hasarlarına ve çıkıklara yol açabilecektir (Şekil 1). ${ }^{[15]}$

Bundan dolayı birçok yazar, 10 yıllık takipte $\% 42,1$ 'e varan başarısızlık oranları olan bu kısıtlı sistemlerin, tekrarlayan instabilite durumunda kullanımının sınırlandırılmasını savunmaktadır. ${ }^{[6,15]}$

Literatürde, tüm bu seçeneklerin başarısız sonuçlarına alternatif olarak dual mobility eklemleşmelerin öne çıktığı görülmektedir. Dual mobility kaplar şu temel prensibe dayanır: Küçük bir kafa, geniş çaplı bir polietilen liner ile eklemlenir; burada femur boynu liner ile temas edene kadar hareket etmekte serbesttir; polietilen ve metal kap arasındaki ikinci bir eklem ise, doğal anatomiye yakın bir kafa boyutu ve hareket açıklığı sağlayarak, daha fazla eklem stabilitesi elde edilmesini sağlar. ${ }^{[16]}$ Özellikle kısıtlı asetabular sistemler ile karşılaştırıldığında, dual mobility eklemler cerrahlar tarafından daha sıklıkla tercih edilir hale gelmiştir. ${ }^{[13,17,18]}$ Ancak dual mobility sistemler kullanıldığında bile, çoklu revizyonlar sonrasında ya da ciddi yumuşak doku kaybı olan olgularda, uzun dönem takiplerde \%15'lere varan çıkıklar olduğu bildirilmiştir. [16]

Asetabulumda kemik kaybı olan revizyon olgularında ilk akla gelen seçenek, metal kafes veya 'jumbo' asetabular kapların sebep olduğu defektin doldurulmasıdır. ${ }^{[19,20]}$ Pelvik devamsızlık varlığında; BurchSchneider ya da Ganz tipi pelvisin çeşitli parçalarını tutan kafesler ve bunların arkasında kalan defektin doldurulması için masif kemik allogreftleri kullanılması önerilir. ${ }^{[21,22]}$ Trabeküler metal, masif defektlerin doldurulmasında şekil verilebilirliği ve iyi osteoentegrasyonu ile son yıllarda giderek daha çok tercih edilen bir malzeme olmuştur ama ne yazık ki ülkemizde, geri ödeme sistemine dâhil olmadığı için, yaygın olarak kullanılamamaktadır. Asetabulumdaki kemik defektlerinde uygulanan bir başka cerrahi teknik de, ilium içerisine uzanım gösteren büyük bir stemin olduğu, konik (cone) ya da LUMIC ${ }^{\circledR}$ tipi asetabular bileşenlerin kullanılmasıdır. ${ }^{[23,24]}$ Son yıllarda, üç boyutlu görüntü işleme ve yazıcı teknolojilerindeki gelişmelere paralel olarak, gerek yapısal stabilitesi gerekse de kalça merkezini anatomik olarak rekonstrükte etme imkânı sunması sayesinde, özel üretim protezler popüler hale gelmeye başlamıştır (Şekil 2).

Benzer şekilde, femoral tarafta yoğun kemik kaybı olduğu durumlarda kullanılan rekonstrüksiyon yöntemleri; impaksiyon greftleme, distalden tutunan press-fit stemler ve tümör protezleridir (megaprotezler) (Şekil 3). ${ }^{[22]}$

$\mathrm{Bu}$ tarz rekonstrüksiyonlardaki en büyük sorun, proksimalde abduktor kolun yapıştığı yerin kaybedilmesi sebebiyle çıkık riskinin çok artması ve aksamanın gelişmesidir. Bu tarz bir cerrahi planlanırken göz önünde bulundurulması gereken bir diğer önemli nokta da, distalde yeterli kemik stoğu bulunması ya da bir diz artroplastisinin varlığıdır. Böyle olgularda yapılması gereken, nadir de olsa kurtarıcı bir yöntem, total femur artroplastisidir.

Yukarıdaki tüm cerrahi tedavi seçenekleri sayesinde, hastaya yeniden fonksiyonel bir kalça sunmak mümkün olmaktadır. Ancak çok küçük bir hasta 

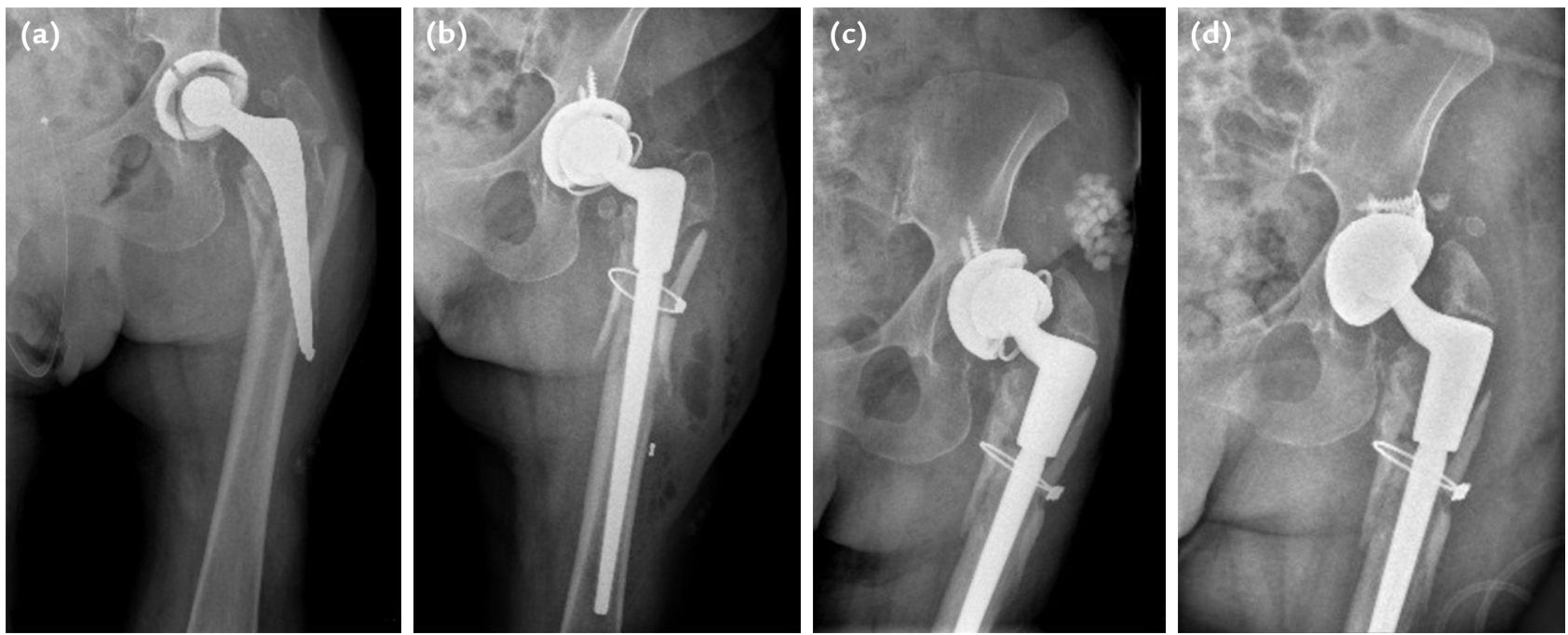

Şekil 1. a-d. Seksen yaşındaki kadın hastaya ait sol kalça direkt grafi görüntülemeleri. İlk cerrahi 17 yıl önce yapılmıştı ve hasta üç yıl önce serebrovasküler olay geçirdiği için sol kalçada adduksiyon kontraktürü varmış; ancak düşme öncesi iki baston yardımıyla yürüyebiliyormuş. Acil servise başvuru sırasında varolan Vancouver tip B3 periprostetik kırık (a). Revizyondan sonra, erken ameliyat sonrası kalça grafisi. Hastada var olan ileri adduksiyon kontraktürü sebebiyle çıkık riskini azaltmak için kısıtlı (constrained) bir asetabular sistem kullanıldı (b). Revizyon cerrahisinden iki yıl sonra, hasta yürüyememe şikâyeti ile başvurduğu zaman çekilen grafide izlenen, kısıtlı asetabular sistemdeki gevşemenin görüntüsü (c). İkinci revizyonda, kısıtlı sistem "dual mobility" ile değiştirildi (d).
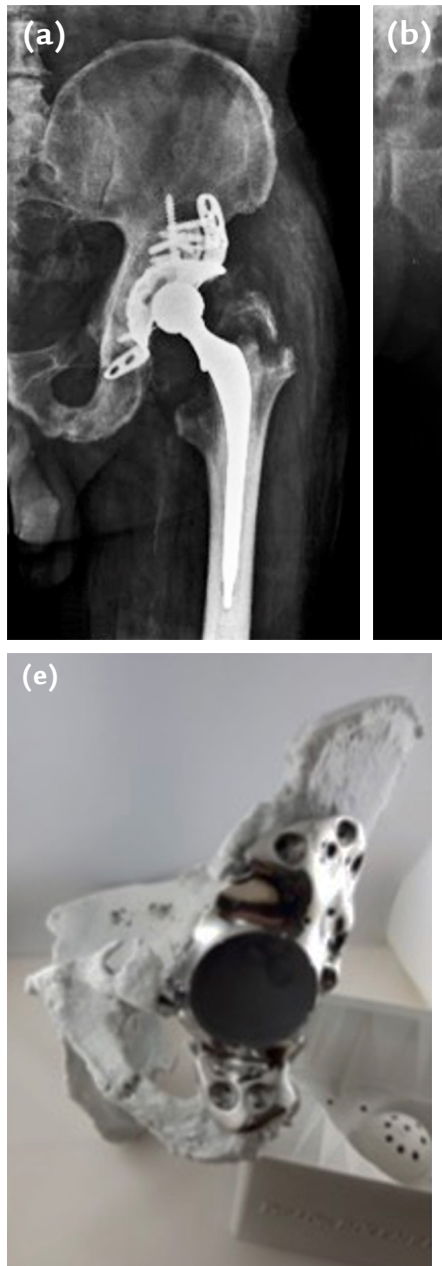
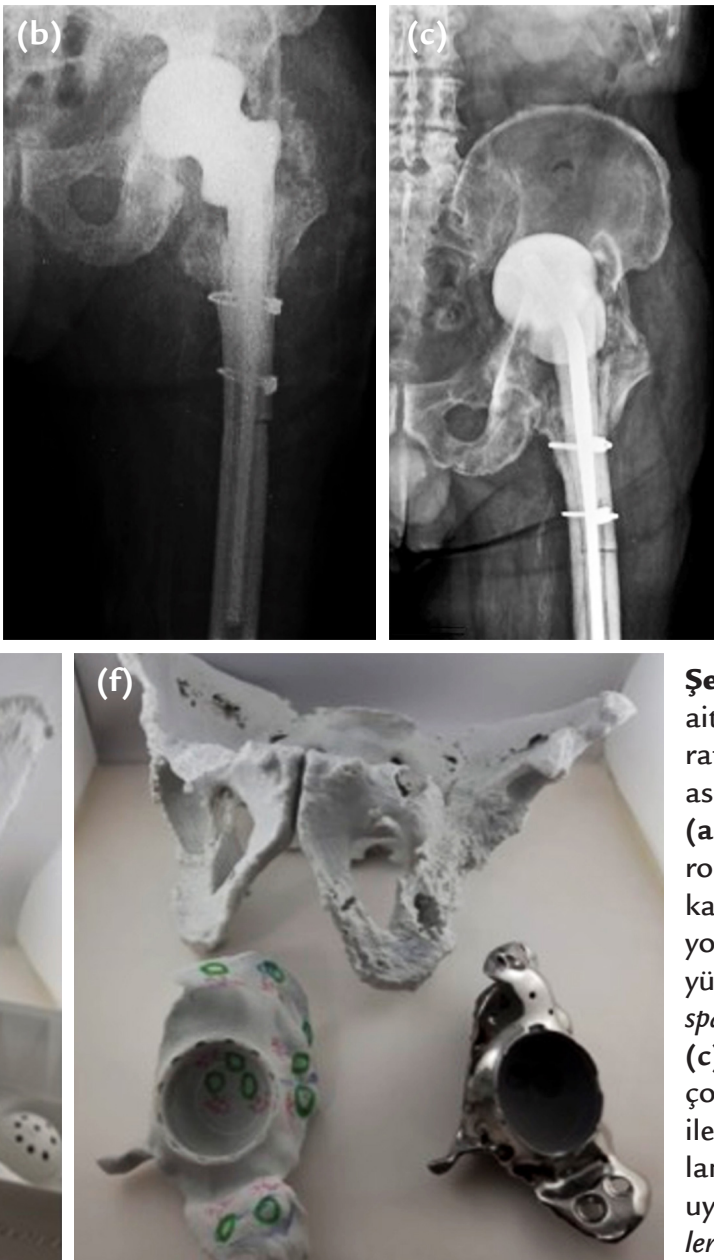

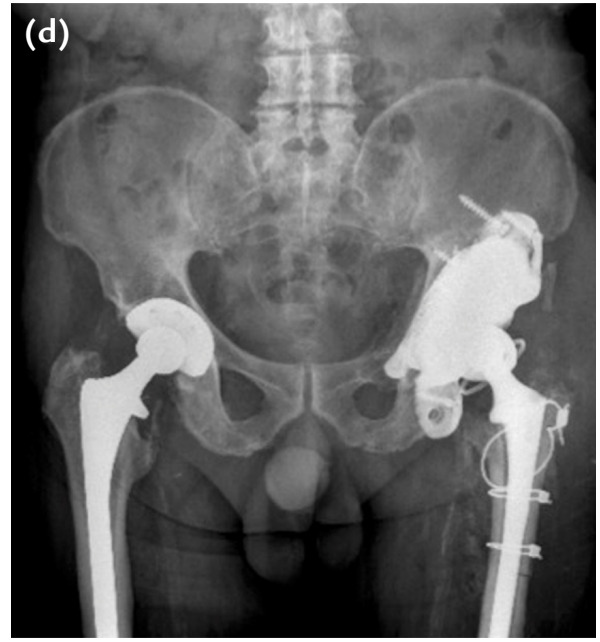

Şekil 2. a-f. Seksen üç yaşındaki erkek hastaya ait olgu grafileri ve özel üretim implant fotoğrafları. Daha önceden, liner aşınması sebebiyle aseptik revizyon yapılmış kalçaya ait direkt grafi (a). Illk revizyon cerrahisinden iki yıl sonra, periprostetik enfeksiyon sebebiyle tüm implantlar çıkartılarak antibiyotik yüklü kalça spacer ile revizyon yapılıyor (b). Hastanın sol alt ekstremitesine yük vermesi sonrası asetabulumda kırık ve kalça spacerının proksimale migrayonu izlenen grafi (c). Hastanın asetabulumdaki kemik defektinin çok büyük olması sebebiyle özel üretim implant ile revizyonu sonrası grafi (d). Özel üretim implantlar ve bunların pelvis kemik modellemesi ile uyumunu gösteren fotoğraflar (e, f). (Olgu grafileri ve fotoğrafları Prof. Dr. Bülent Erdemli'ye aittir.) 

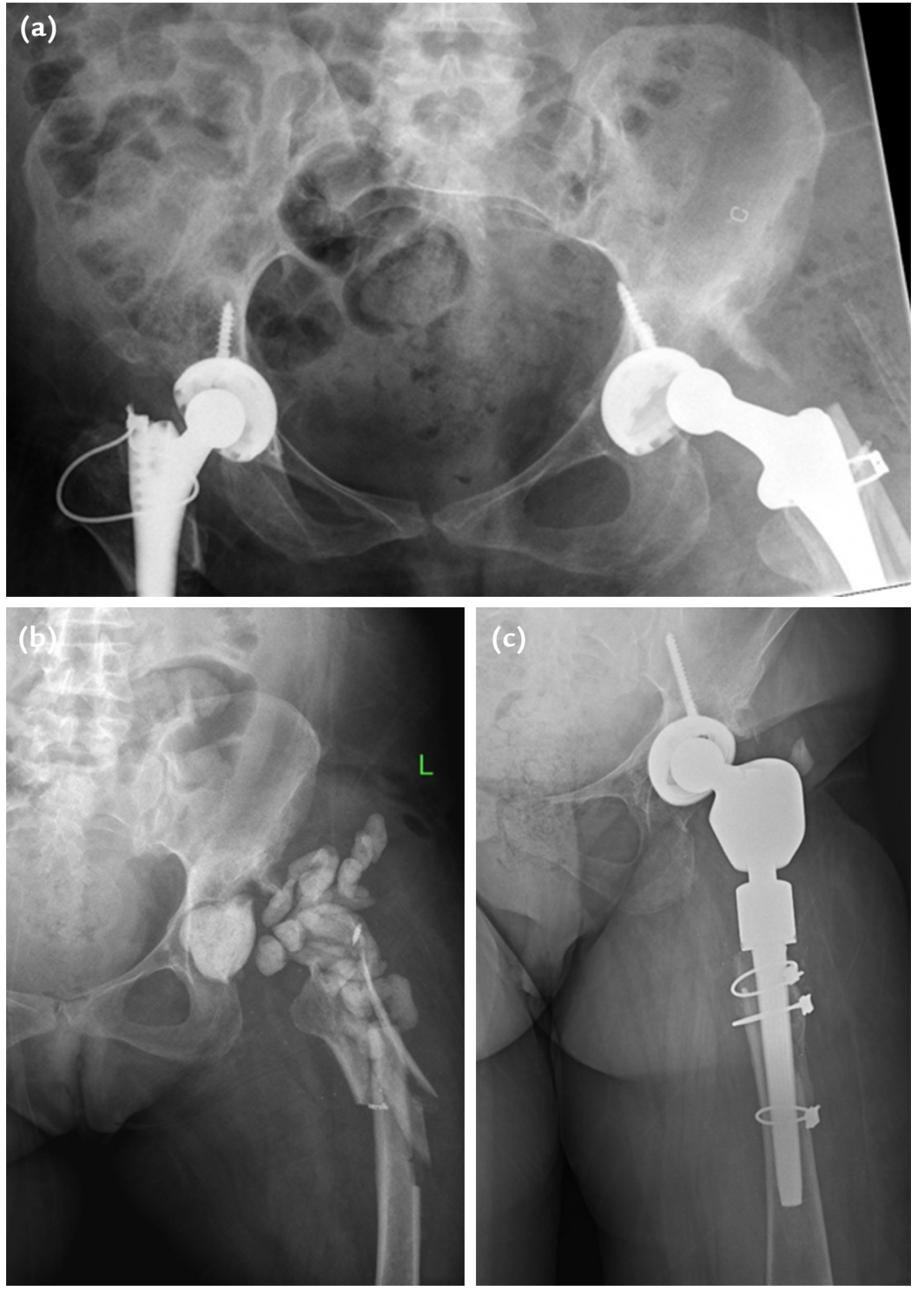

Şekil 3. a-c. Altmış dokuz yaşındaki kadın hastaya ait olgu grafileri ve megaprotez fotoğrafları. Dış merkezde total kalça artroplastisi (TKA) yapılmış ve sol kalçasında tekrarlayan çıkık izlenen hastanın pelvis anteroposterior (AP) grafisi (a). Hasta, kliniğimize ilk başvurusu sırasında, çoklu cerrahi sonrası gelişen protez enfeksiyonu ve periprostetik kırıklar sebebiyle sol alt ekstremiteye yük veremeyecek durumdaydı (b). Sol proksimal femurdaki geniş kemik kaybı sebebiyle proksimal femur replasmanı sağlayan megaprotez ile kalçanın rekonstrüksiyonu sonrası grafisi (c). (Olgu grafileri Doç. Dr. Kerem Başarır'a aittir.) grubunda, yukarıda anlatılan işlemler bile yeterli gelmez ve kurtarıcı işlem olarak son çare, sarsak kalça eklemi (girdlestone) uygulanabilir. Sarsak kalça ekleminin en önemli dezavantajı, ortaya çıkan bacak uzunluğu farkıdır. ${ }^{[25]}$ Ancak zaman içinde proksimal femur yukarı doğru hareket eder, ölü boşluk azalır ve hasta yeni kalça biyomekaniği ile yürümeye alışır. ${ }^{[22]}$

\section{SONUÇ}

Modern teknikler ile primer kalça artroplastisinde elde edilen başarılı sonuçlara rağmen; revizyon ve başarısızıı, ortopedistler ve sağlık sistemi için ciddi bir sorun oluşturmaya devam etmektedir. ${ }^{[2,10,26]}$ Cerrahi teknik ve implant tasarımlarındaki iyileştirmeler ve cerrahi öncesinde hastanın risk değerlendirilmesi gibi birçok atılıma rağmen; revizyon kalça artroplastileri halen artış göstermektedir. ${ }^{[5,10]}$ Revizyon ihtiyacını azaltmak ve artroplasti olgularının sağkalımını artırmak için, altta yatan etkenlere yönelik daha ayrıntılı çalışmalar yapılması önemli bir gerekliliktir.

\section{Teşekkür ve Katkı}

Olgularına ait grafi ve fotoğrafları paylaşarak bu yayında kullanmama izin veren Ankara Üniversitesi Tıp Fakültesi, Ortopedi ve Travmatoloji Ana Bilim Dalı Öğretim Üyeleri Prof. Dr. Bülent Erdemli ve Doç. Dr. Kerem Başarır'a teşekkürlerimi sunarım. 


\section{KAYNAKLAR}

1. Yu S, Saleh H, Bolz N, Buza J, lorio R, Rathod PA, Schwarzkopf R, Deshmukh AJ. Re-revision total hip arthroplasty: Epidemiology and factors associated with outcomes. J Clin Orthop Trauma 2020;11(1):43-6. Crossref

2. Kurtz S, Ong K, Lau E, Mowat F, Halpern M. Projections of primary and revision hip and knee arthroplasty in the United States from 2005 to 2030. J Bone Joint Surg Am 2007;89(4):780-5. Crossref

3. Khatod M, Cafri G, Inacio MCS, Schepps AL, Paxton EW, Bini SA. Revision total hip arthoplasty: factors associated with rerevision surgery. J Bone Joint Surg Am 2015;97(5):359-66. Crossref

4. Ong KL, Lau E, Suggs J, Kurtz SM, Manley MT. Risk of Subsequent Revision after Primary and Revision Total Joint Arthroplasty. Clin Orthop Relat Res 2010;468(11):3070-6. Crossref

5. Gwam CU, Mistry JB, Mohamed NS, Thomas M, Bigart KC, Mont MA, Delanois RE. Current Epidemiology of Revision Total Hip Arthroplasty in the United States: National Inpatient Sample 2009 to 2013. J Arthroplasty 2017;32(7):2088-92. Crossref

6. Wegrzyn J, Tebaa E, Jacquel A, Carret J-P, Béjui-Hugues J, Pibarot V. Can Dual Mobility Cups prevent Dislocation in All Situations After Revision Total Hip Arthroplasty? J Arthroplasty 2015;30(4):631-40. Crossref

7. Jafari SM, Coyle C, Mortazavi SMJ, Sharkey PF, Parvizi J. Revision hip arthroplasty: Infection is the most common cause of failure. Clin Orthop Relat Res 2010;468(8):204651. Crossref

8. Lübbeke A, Roussos C, Barea C, Köhnlein W, Hoffmeyer P. Revision total hip arthroplasty in patients 80 years or older. J Arthroplasty 2012;27(6):1041-6. Crossref

9. Springer BD, Fehring TK, Griffin WL, Odum SM, Masonis JL. Why revision total hip arthroplasty fails. Clin Orthop Relat Res 2009;467(1):166-73. Crossref

10. Bozic KJ, Kamath AF, Ong K, Lau E, Kurtz S, Chan V, Vail TP, Rubash H, Berry DJ. Comparative Epidemiology of Revision Arthroplasty: Failed THA Poses Greater Clinical and Economic Burdens Than Failed TKA. Clin Orthop Relat Res 2014;473(6):2131-8. Crossref

11. Petis SM, Abdel MP, Perry KI, Mabry TM, Hanssen AD, Berry DJ. Long-term results of a 2-stage exchange protocol for periprosthetic joint infection following total hip arthroplasty in 164 hips. J Bone Joint Surg Am 2019;101(1):74-84. Crossref

12. Zahar A, Klaber I, Gerken A-M, Gehrke T, Gebauer M, Lausmann C, Citak M. Ten-Year Results Following One-Stage Septic Hip Exchange in the Management of Periprosthetic Joint Infection. J Arthroplasty 2019;34(6):1221-6. Crossref

13. Van Heumen M, Heesterbeek PJC, Swierstra BA, Van Hellemondt GG, Goosen JHM. Dual mobility acetabular component in revision total hip arthroplasty for persistent dislocation: no dislocations in 50 hips after $1-5$ years. J Orthopaed Traumatol 2015;16(1):15-20. Crossref
14. Caton JH, Ferreira A. Dual-mobility cup: a new French revolution. Int Orthop 2017;41(3):433-7. Crossref

15. Guyen O, Pibarot V, Vaz G, Chevillotte C, Béjui-Hugues J. Use of a dual mobility socket to manage total hip arthroplasty instability. Clin Orthop Relat Res 2009;467(2):465-72. Crossref

16. Unter Ecker N, Kocaoğlu H, Zahar A, Haasper C, Gehrke T, Citak M. What Is the Dislocation and Revision Rate of Dualmobility Cups Used in Complex Revision THAs? Clin Orthop Relat Res 2021;479(2):280-5. Crossref

17. Mertl P, Combes A, Leiber-Wackenheim F, Fessy MH, Girard $\mathrm{J}$, Migaud $\mathrm{H}$. Recurrence of dislocation following total hip arthroplasty revision using dual mobility cups was rare in 180 hips followed over 7 years. HSS J 2012;8(3):251-6. Crossref

18. Mohammed R, Hayward K, Mulay S, Mulay S, Bindi F, Wallace M. Outcomes of dual-mobility acetabular cup for instability in primary and revision total hip arthroplasty. J Orthopaed Traumatol 2015;16(1):9-13. Crossref

19. Hsu C-C, Hsu C-H, Yen S-H, Wang J-W. Use of the BurchSchneider cage and structural allografts in complex acetabular deficiency: 3- to 10-year follow up. Kaohsiung J Med Sci 2015;31(10):540-7. Crossref

20. Schmidt-Braekling T, Sieber D, Gosheger G, Theil JC, Moellenbeck B, Andreou D, Dieckmann R. Dislocation rates with combinations of antiprotrusio cages and dual mobility cups in revision cases: Are we safe? PLoS One 2019;14(2):111. Crossref

21. Schneider L, Philippot R, Boyer B, Farizon F. Revision total hip arthroplasty using a reconstruction cage device and a cemented dual mobility cup. Orthop Traumatol Surg Res 2011;97(8):807-13. Crossref

22. Uchiyama K, Inoue G, Takahira N, Takaso M. Revision total hip arthroplasty - Salvage procedures using bone allografts in Japan. J Orthop Sci 2017;22(4):593-600. Crossref

23. Bus MPA, Szafranski A, Sellevold S, Goryn T, Jutte PC, Bramer JAM, Fiocco M, Streitbürger A, Kotrych D, van de Sande MAJ, Dijkstra SPD. LUMiC $®$ Endoprosthetic Reconstruction After Periacetabular Tumor Resection: Short-term Results. Clin Orthop Relat Res 2017;475(3):686-95. Crossref

24. Issa S-P, Biau D, Leclerc P, Babinet A, Hamadouche M, Anract P. Stemmed acetabular cup as a salvage implant for revision total hip arthroplasty with Paprosky type IIIA and IIIB acetabular bone loss. Orthop Traumatol Surg Res 2020;106(3):589-96. Crossref

25. Gebert C, Wessling M, Hoffmann C, Roedl R, Winkelmann W, Gosheger G, Hardes J. Hip transposition as a limb salvage procedure following the resection of periacetabular tumors. J Surg Oncol 2011;103(3):269-75. Crossref

26. Bozic BKJ, Kurtz SM, Lau E, Ong K, Vail TP, Berry DJ. The Epidemiology of Revision Total Hip Arthroplasty in the United States. J Bone Joint Surg Am 2009;91(1):128-33. Crossref 